monitoring was negative for seizures. Transcranial doppler monitoring over the next few days demonstrated worsening velocities, with a repeat angiogram confirming severe focal segmental irregularities isolated to the right anterior territory. The surgical and stenting site remained widely patent. Her vasospasm was successfully treated with intra-arterial verapamil with improvement in vessel caliber and clinical symptoms.

Conclusions Post carotid revascularization cerebral vasoconstriction may be an unusual cause of clinical worsening after revascularization for chronic carotid stenosis or occlusion. Intra-arterial treatment with verapamil can be a safe and effective mode of therapy.

Disclosures L. Sheikhi: None. J. Tsai: None. M. Bain: 2; C; Stryker. 4; C; Rebound Therapeutics. G. Toth: None.

\section{E-087 HYBRID OPEN AND ENDOVASCULAR APPROACH FOR OCCIPITAL ARTERIOVENOUS FISTULA WITH COMPLETE OBLITERATION: TECHNICAL NOTE}

1J Lavie, ${ }^{2} \mathrm{M}$ Mathkour*, ${ }^{1} \mathrm{P}$ Gulotta, ${ }^{3} \mathrm{G}$ Vidal, ${ }^{1} \mathrm{~J}$ Milburn, ${ }^{2} \mathrm{E}$ Valle-Giler. ${ }^{1}$ Radiology, Ochsner Medical Center, New orleans, LA; ${ }^{2}$ Neurosurgery, Tulane Medical Center/Ochsner Medical Center, New orleans, LA; ${ }^{3}$ Neurology, Ochsner Medical Center, New orleans, LA

10.1136/neurintsurg-2019-SNIS.162

Background Dural arteriovenous fistulas (DAVFs) are acquired abnormal shunting connexions between the meningeal arteries and dural sinus or cortical veins and are an important cause of intracranial hemorrhage. Lesions with retrograde cortical venous reflux carry a higher risk of bleeding and endovascular embolization is currently the first-line treatment for these lesions. We present a case of DAVF with cortical venous reflux not amenable to conventional transarterial or transvenous endovascular embolization treated with direct surgical access to the occipital artery and Onyx embolization.

Method A 67-year-old man with a history of seizure disorder who presented with a tonic-clonic seizure one month after stopping his antiepileptics. Neurologic examination was unremarkable aside from diminished hearing on the right. The patient presented for neurosurgical evaluation due to imaging findings performed for work-up of his most recent seizure. Non-contrast head CT was unremarkable. CT and MR angiogram were performed, demonstrating prominent cortical veins overlying the right temporal convexity, suspicious for dural AV fistula or arteriovenous malformation. Digital subtraction angiography confirmed a dural AV fistula with arterial supply from the right occipital and middle meningeal arteries and shunting to the transverse sinus with multiple dilated cortical veins, compatible with a Borden 3, Cognard 4 lesion. In addition, there was a type 3 aortic arch with an extremely tortuous right common carotid artery, precluding selective access to the external carotid artery by either transfemoral or transradial approach. Due to the inability to access the right external carotid artery via conventional endovascular approaches, we elected to perform a hybrid procedure with surgical cutdown for direct access to the right occipital artery for Onyx embolization.

Result Using ultrasound guidance, the right occipital artery was tracked over the posterior nuchal line. A linear incision was designed over it and the artery was exposed and catheterized with a short $5 \mathrm{~F}$ sheath. Vessels loops were used to secure the sheath to the artery. An onyx compatible microcatheter was used to access the nidus of the fistula and under direct fluoroscopy the fistula was embolized. A Post-embolization angiogram from the right occipital artery demonstrated no residual filling of the fistula. The postoperative was unremarkable and follow up angiogram at 6 months shows complete obliteration.

Conclusion A variety of hybrid open and endovascular approaches have been described and the majority describes approaches involve a combination of a craniotomy followed by Onyx or NBCA embolization after direct cannulation of the artery or vein. In this case, we report a combined surgical and endovascular approach for Borden III DAVF that was inaccessible to standard endovascular approaches due to tortuous carotid anatomy via a direct cut-down. Direct cannulation of the occipital artery provided an elegant access to the fistula distal to the tortuous carotid anatomy with minimally invasive surgery without requiring a craniotomy, and a large volume of Onyx was able to be injected via the prominent occipital artery. Direct cannulation of feeding vessels to dural AVF represent a treatment option in patients that are not good candidates for conventional endovascular or open approaches.

Disclosures J. Lavie: None. M. Mathkour: None. P. Gulotta: None. G. Vidal: None. J. Milburn: None. E. Valle-Giler: None.

\section{E-088 PSYCHOLOGICAL IMPACT OF PULSATILE TINNITUS IN THE US POPULATION}

${ }^{1} \mathrm{E}$ Smith*, ${ }^{2} \mathrm{M}$ Amans, ${ }^{3} \mathrm{~K}$ Meisel, ${ }^{4} \mathrm{D}$ McCoy. ${ }^{1}$ Radiology, Medical College of Wisconsin, Milwaukee, W/; ${ }^{2}$ Neurointerventional Radiology, University of California, San Francisco, San Francisco, $C A ;{ }^{3}$ Neurology, University of California, San Francisco, San Francisco, $C A_{i}$ ${ }^{4}$ Radiology, University of California, San Francisco, San Francisco, CA

\subsection{6/neurintsurg-2019-SNIS.163}

Introduction/Purpose Pulsatile tinnitus (PT) is the auditory perception of a rhythmic, cardiac-synchronized, 'whooshing' type of sound in the absence of an external source that affects 3-5 million Americans. In addition, several of the anomalies that cause PT can have serious sequelae such as ischemic stroke, blindness, or intracranial hemorrhage. In addition, it is common for patients to describe an inability to continue to function in their families and in society due to the psychological impact of PT (which may be depression, anxiety, or a combination). In our experience, treating the underlying cause of PT not only mitigates the risks of the vascular anomaly, but also the patients' psychiatric illness. However, the prevalence of depression and anxiety in the PT population is unknown. The purpose of this study was to quantify the prevalence of depression and anxiety in the United States PT population, as well as identify relationships between patient characteristics, effects of PT on various aspects of life, depression, and anxiety.

Materials and methods Local IRB approval was obtained to survey the pulsatile tinnitus community. The survey used the validated Tinnitus Functional Index (TFI) to determine the severity of the PT condition (intrusive, sense of control, cognitive, sleep, auditory, relaxation, quality of life, emotional). In addition, the PHQ-9 and GAD-7 was used to obtain the prevalence of concurrent depression and anxiety, respectively. Logistic multiple regression analysis was performed for determining the associations of clinical and demographic variables with TFI total score. Additionally, PHQ9 and GAD7 scores (binarized to indicate depression/anxiety) were modeled with TFI total score as the main exposure 
variable with possible covariates in multiple regression modeling.

Results The prevalence of moderate to severe depression in the PT population was $41.2 \%$. Multiple regression to predict PHQ-9 diagnosed depression based on TFI total score, clinical variables, and demographics showed a significant odds ratio for TFI (OR: 1.084, CI: 1.065-1.096, p<0.001). The increase of sub-scores for quality of life interference and emotionality showed significant increases in odds for depression (OR: 1.031, CI: 1.016-1.046, p<0.001; OR: 1.042, CI: 1.027$1.058, \mathrm{p}<0.001)$. Unemployed vs. employed and laterality were also associated with an increased odds of depression, and age with decreased odds while controlling for tinnitus score.

Moderate to severe anxiety was present in $43.1 \%$. Multiple regression analysis showed TFI was associated with increased of odds for anxiety (OR: 1.056, CI: 1.043-1.070, p<0.001). TFI sub-scores for emotionality (OR: 1.043, $\mathrm{p}<0.001$ ), quality of life (OR: 1.019, $\mathrm{p}<0.005)$, and cognitive interferences (OR: $0.983, p=0.015$ ) were found to be significantly associated with anxiety scores.

Conclusion The estimated prevalence of moderate to severe depression and anxiety in the US PT population is $41.2 \%$ and $43.1 \%$, respectively, which is higher than a recent systematic review of depression in tinnitus suggesting a depression prevalence of 33\%. TFI score was significantly associated with both depression and anxiety. Similarly, more granular sub-scores of the TFI elucidate how PT affects the psychological health of these patients.

Disclosures E. Smith: None. M. Amans: 1; C; This work was funded by an NIH grant. 2; C; Consultant for Covidien and Stryker. K. Meisel: None. D. McCoy: None.

\section{E-089 EARLY TRANSCRIPTIONAL REGULATION OF TENASCIN-C ATTENUATES POST-STROKE BRAIN DAMAGE}

${ }^{1} \mathrm{~K}$ Johnson*, ${ }^{2} \mathrm{~B}$ Chelluboina. ${ }^{1}$ Neurosurgery, University of Illinois, Peoria, IL; ${ }^{2}$, Neurosurgery, University of Wisconsin School of Medicine, Madison, WI

10.1136/neurintsurg-2019-SNIS. 164

Background Stroke induced alterations in the Extracellular matrix play a vital role in mediating acute pathogenesis as well as in post-stroke recovery. Tenascin-C (TNC), an adhesion-modulating extracellular matrix glycoprotein, its upregulation in adults is linked to vascular diseases. Recently, an elevated blood level of TNC in large artery atherosclerotic stroke patients and its association with post-stroke inflammation is reported. Based on the literature and our recent research investigation, we hypothesize that specific suppression of TNC immediately after ischemic stroke by using a non-viral shRNA attenuated the post-stroke acute brain damage.

Methods In order to attain our objectives, we utilized young adult male Sprague-Dawley rats. Selected animal groups were subjected to two-hour middle cerebral artery occlusion followed by three-day reperfusion with or without TNCshRNA treatment immediately after reperfusion. We performed various techniques including TTC, Realtime-PCR, and immunoblot and immunofluorescence analysis in order to investigate the effect of our treatments and the molecular mechanisms.
Results Our results clearly demonstrated that TNC is persistently upregulated after ischemic stroke. Specific regulation of TNC immediately after ischemia inhibited the TNC levels and significantly reduced the extent of brain damage. TNC inhibition after stroke modulated the expression of excitatory amino acid transporters and tight junction proteins. Further, Early inhibition of TNC after stroke regulated the expression of key inflammatory mediators such as Toll-Like Receptor-4 and Tumor Necrosis Factor- $\alpha$ and Matrix metalloproteinase-9.

Conclusions Based on our results we conclude that TNC mediated several acute ischemic stroke pathogenic mechanisms. Transcriptional inactivation of TNC regulated the post-stroke alterations in excitatory amino acid transporters, tight junction proteins, and inflammatory mediators and thus attenuated the post-stroke brain damage.

Disclosures K. Johnson: None. B. Chelluboina: None.

\section{E-090 GEOGRAPHIC SERVICE DELIVERY FOR ENDOVASCULAR CLOT RETRIEVAL: USING DISCRETE EVENT SIMULATION TO OPTIMISE RESOURCES}

${ }^{1} \mathrm{Y}$ Ren ${ }^{*},{ }^{2} \mathrm{M}$ Phan, ${ }^{2} \mathrm{~J}$ Maingard, ${ }^{2} \mathrm{C}$ Seah, ${ }^{2} \mathrm{~J} \mathrm{Wu},{ }^{3} \mathrm{P}$ Luong, ${ }^{2} \mathrm{D}$ Shell, ${ }^{4} \mathrm{M}$ Burney, ${ }^{2} \mathrm{~K}$ Zhou, ${ }^{1} \mathrm{~A}$ lamanna, ${ }^{5} \mathrm{H}$ Kok, ${ }^{2} \mathrm{R}$ Chandra, ${ }^{6} \mathrm{~A}$ Jhamb, ${ }^{1} \mathrm{~V}$ Thijs, ${ }^{1} \mathrm{D}$ Brooks, ${ }^{1} \mathrm{H}$ Asadi. ${ }^{1}$ A ustin health, Melbourne, AUSTRALIA; ${ }^{2}$ Monash health, Melbourne, Australia; ${ }^{3}$ RMIT, Melbourne, Australia; ${ }^{4}$ Deloitte Digital, Sydney, Australia; ${ }^{5}$ Northern Health, Melbourne, Australia; ${ }^{6}$ St Vincent's Hospital, Melbourne, Australia

\subsection{6/neurintsurg-2019-SNIS. 165}

Background Endovascular thrombectomy is the standard of care for acute ischaemic stroke caused by large vessel occlusion. Reducing stroke symptom onset to reperfusion time is associated with improved functional outcomes. In this study we aimed to develop a computational model to predict and identify time-related outcomes of community stroke calls within an area based on variable parameters.

Method A model to simulate and predict EVT service delivery at capable hospital within a geographic area, city or state was designed using SimPy, a Python based discrete event simulation framework. Geolocation data defined by the user as well as that used by the model is sourced using the Google Maps Application Programming Interface (API) and GeoPy. Variables are customized by the user based on their local environment to provide more acute prediction.

Result This algorithm can predict the delay between the time that emergency services are notified of a potential stroke and cerebral reperfusion using EVT at capable hospital. Factors including area size and population, number of EVT capable hospitals, number of available angiography machines, availability of ambulances and time of patient can be adjusted to observe the effect of modifying each parameter input.

Conclusion This model, made available as an open source web-based application, provides a mean of predicting resource utilisation and wait times for stroke service delivery planning. By modifying parameter inputs, the delivery and coordination of a stroke service within an area, city or state may be optimised and aid the development of new EVT service protocols. Disclosures Y. Ren: None. M. Phan: None. J. Maingard: None. C. Seah: None. J. Wu: None. P. Luong: None. D. Shell: None. M. Burney: None. K. Zhou: None. A. lamanna: None. H. Kok: None. R. Chandra: None. A. Jhamb: None. V. Thijs: None. D. Brooks: None. H. Asadi: None. 